\title{
Amorphous Silicon Based Neutron Detector
}

\author{
Final Technical Report
}

Submitted to

\section{US Department of Energy}

under SBIR Phase I contract DE-FG02-03ER83718

July 21, 2003-April 20, 2004

Submitted by: $\quad$ Midwest Optoelectronics, LLC

Address:

2600 Dorr Street

Toledo, Ohio 43607

Ph: (419) 724-3709

Fax: (419) 531-8465

Dr. Liwei Xu, President, MWOE \& Principal Investigator

Ph: (419) 215-8583; Email: xu@mwoe.com

Mr. Stanley Rubini, CEO, MWOE

Ph: (419) 283-7433; Email: rubini@mwoe.com

In collaboration with: University of Toledo

Dr. Xunming Deng: (419) 530-4782; dengx@physics.utoledo.edu

Dr. Chandan Das: (419) 530-4905; cdas@physics.utoledo.edu

Dr. Song Cheng: (419) 530-4788; scheng@physics.utoledo.edu

Oak Ridge National Laboratory

Dr. David Holcomb: (865) 576-7889; holcombde@ornl.gov

Mr. Joseph Williams: (865) 576-8285; williamsja@ornl.gov 


\section{Amorphous Silicon Based Neutron Detector}

\section{Summary}

Various large-scale neutron sources already build or to be constructed, are important for materials research and life science research. For all these neutron sources, neutron detectors are very important aspect. However, there is a lack of a high-performance and low-cost neutron beam monitor that provides time and temporal resolution.

The objective of this SBIR Phase I research, collaboratively performed by Midwest Optoelectronics, LLC (MWOE), the University of Toledo (UT) and Oak Ridge National Laboratory (ORNL), is to demonstrate the feasibility for amorphous silicon based neutron beam monitors that are pixilated, reliable, durable, fully packaged, and fabricated with high yield using low-cost method.

During the Phase I effort, work as been focused in the following areas:

1) Deposition of high quality, low-defect-density, low-stress a-Si films using very high frequency plasma enhanced chemical vapor deposition (VHF PECVD) at high deposition rate and with low device shunting;

2) Fabrication of $\mathrm{Si} / \mathrm{SiO}_{2} / \mathrm{metal} / \mathrm{p} / \mathrm{i} / \mathrm{n} / \mathrm{metal} / \mathrm{n} / \mathrm{i} / \mathrm{p} / \mathrm{metal} / \mathrm{SiO}_{2} /$ device for the detection of alpha particles which are daughter particles of neutrons through appropriate nuclear reactions; and

3) Testing of various devices fabricated for alpha and neutron detection;

As the main results:

- High quality, low-defect-density, low-stress a-Si films have been successfully deposited using VHF PECVD on various low-cost substrates;

- Various single-junction and double junction detector devices have been fabricated;

- The detector devices fabricated have been systematically tested and analyzed.

- Some of the fabricated devices are found to successfully detect alpha particles.

Further research is required to bring this Phase I work beyond the feasibility

demonstration toward the final prototype devices.

The success of this project will lead to a high-performance, low-cost, X-Y pixilated neutron beam monitor that could be used in all of the neutron facilities worldwide. In addition, the technologies developed here could be used to develop X-ray and neutron monitors that could be used in the future for security checks at the airports and other critical facilities.

The project would lead to devices that could significantly enhance the performance of multi-billion dollar neutron source facilities in the US and bring our nation to the forefront of neutron beam sciences and technologies which have enormous impact to materials, life science and military research and applications. 


\section{Introduction}

This report covers the development of neutron detector for steady state and pulsed neutron scattering facilities under SBIR contract \#DE-FG02-03ER83718.

\section{Technical approach}

In the development of neutron detector the first approach was to develop a alpha particle detector since alpha particles are daughter particle of ${ }^{10} \mathrm{~B}(\mathrm{n}, \alpha){ }^{7} \mathrm{Li}$ reaction. With a coat of ${ }^{10} \mathrm{~B}$ on alpha detector, it works in principle as a neutron detector.

\section{Development of alpha particle detector}

The development of alpha particle detector includes both the optimization of a-Si thin films properties for device application and optimization of the device itself. In the process of development of alpha particle detector, we focused our activities on (a) development of a-Si:H material as active layer of the device, (b) fabrication of the proposed device structure and (c) detection of alpha particles.

\section{(a) Development of a-Si:H thin film material}

With the prime demand of a high rate deposition and a very thick film $(20-30 \mu \mathrm{m})$ of a-Si:H as active (absorber) layer for the detector, we employed very high frequency (VHF: $70 \mathrm{MHz}$ ) in our PECVD system and successfully obtained the required intrinsic type a-Si:H material.

We optimized the VHF power and flow of hydrogen $\left(\mathrm{H}_{2}\right)$ and disilane $\left(\mathrm{Si}_{2} \mathrm{H}_{6}\right)$ in deposition process. We used $9 \mathrm{sccm}$ and $54 \mathrm{sccm}$ flow of disilane and hydrogen respectively at a power of 45 Watt where we achieved the best deposition rate as high as $20 \AA / \mathrm{s}$. The optimization of deposition rate and thickness of the film has been based on other concerns as the sticking problem of the thick film on a foreign substrate, choice of substrate and the post deposition cooling rate of the sample. To optimize and investigate the sticking factor we employed different types of substrates and taken post-deposition measure to prevent mechanical peeling off of the a-Si layer from the substrate. We used stainless steel (SS), glass and c-Si wafer as three different substrates of which both the front and back sides of the c-Si was been tried. We also allowed the samples to cool down at a low rate after deposition of thick i-layer by VHF PECVD technique. A highly thick a-Si:H layer deposited at high rate causes huge compressive stress in the film and this stress relaxes during the cooling down, effectively peeling the film off the surface. Slow cooling rate retards the relaxation rate which prevents the peeling off. We optimized the cooling rate and at a cooling rate of $50^{\circ} \mathrm{C} / 60 \mathrm{~min}$, we achieved a good sticking for the most of the samples prepared during the other optimization runs. The sticking of the films was observed to be the best on SS followed by c-Si front, glass and c-Si back surfaces.

This optimization process enabled us to achieve a proper intrinsic layer of a-Si:H as the active layer of $\mathrm{p}-\mathrm{i}-\mathrm{n}$ diode type device structure. In table 1 , the deposition 
conditions and some results on i-layers are shown. For the $\mathrm{p}$ and $\mathrm{n}$ type doped layers, we also used previously optimized conditions for the deposition process of $\mathrm{p}-\mathrm{i}-\mathrm{n}$ diodes.

Table 1: Deposition conditions and some results on i-layer of the p-i-n structure.

\begin{tabular}{|l|c|c|c|c|c|c|}
\hline $\begin{array}{c}\text { Sample } \\
\text { name }\end{array}$ & $\begin{array}{c}\text { Thickness } \\
\text { on glass } \\
(\mu \mathrm{m})\end{array}$ & $\begin{array}{c}\text { Deposition } \\
\text { rate }(\AA / \mathrm{s})\end{array}$ & $\begin{array}{c}\mathrm{Si}_{2} \mathrm{H}_{6} \\
(\mathrm{sccm})\end{array}$ & $\begin{array}{c}\mathrm{H}_{2} \\
(\mathrm{sccm})\end{array}$ & $\begin{array}{c}\text { Power } \\
(\text { Watt })\end{array}$ & Cooling Time \\
\hline GD1096 & 5.5 & 10 & 3 & 18 & 20 & $2 \mathrm{Hr}$ \\
\hline GD1098 & 12 & 12 & 4.5 & 27 & 30 & $2 \mathrm{Hr}$ \\
\hline GD1099 & 12 & 8 & 3 & 18 & 20 & $1 \mathrm{Hr}$ \\
\hline GD1100 & 10 & 10 & 4.5 & 27 & 45 & $2 \mathrm{Hr}$ \\
\hline GD1101 & 21 & 20 & 9 & 54 & 45 & $2 \mathrm{Hr}$ \\
\hline
\end{tabular}

\section{(b) Fabrication of the proposed device structure}

The activities on fabrication of the device structure are reported according to the sequence (following with sample numbers) of the fabrication and characterization processes. The problems regarding fabrication are addressed; their solutions and results are discussed accordingly.

GD1096:

The device consists of a substrate, $n$-i-p silicon layers acting as diode and a top contact layer. For device fabrication we used c-Si (front surface, polished) and SS as substrates in our first batch of attempts considering the sticking factor in mind. Initially we tried to fabricate device of small size (circular area $0.25 \mathrm{~cm}^{2}$ ) on both substrates. We optimized the doped layer condition following our previous alpha particle detection from sample GD671. A schematic diagram and a photograph of the device are shown in Figure 1 and Figure 2 respectively.

\begin{tabular}{|c|c|}
\hline $\begin{array}{c}\text { TCO/Gold } \\
\text { grid }\end{array}$ & $\begin{array}{c}\text { TCO/Gold } \\
\text { grid }\end{array}$ \\
\hline p - a-Si:H \\
\hline i - a-Si:H \\
\hline n - a-Si:H \\
\hline c-Si substrate \\
\hline
\end{tabular}

Fig. 1 Schematic diagram of sample \# GD1096 with TCO/gold top contact grids, fabricated on c-Si substrate. 


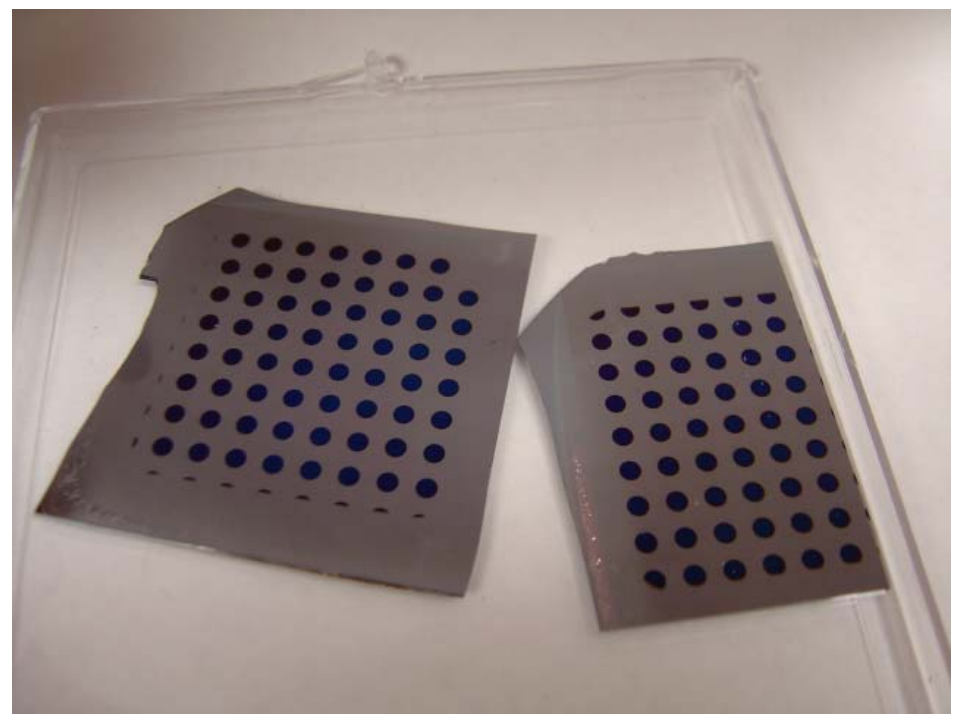

Fig. 2 Photograph of sample \# GD1096 with small area $\left(0.05 \mathrm{~cm}^{2}\right)$ devices fabricated on c-Si substrate.

We measured the leakage current under reverse bias for each of the devices and found high leakage current under reverse bias at $-200 \mathrm{~V}$. For the devices on SS, the leakage current is much higher and the yield is poorer also. In this sample we i-layer thickness of $5.5 \mu \mathrm{m}$. A graph of leakage current at different reverse bias voltage is shown in Figure 3.

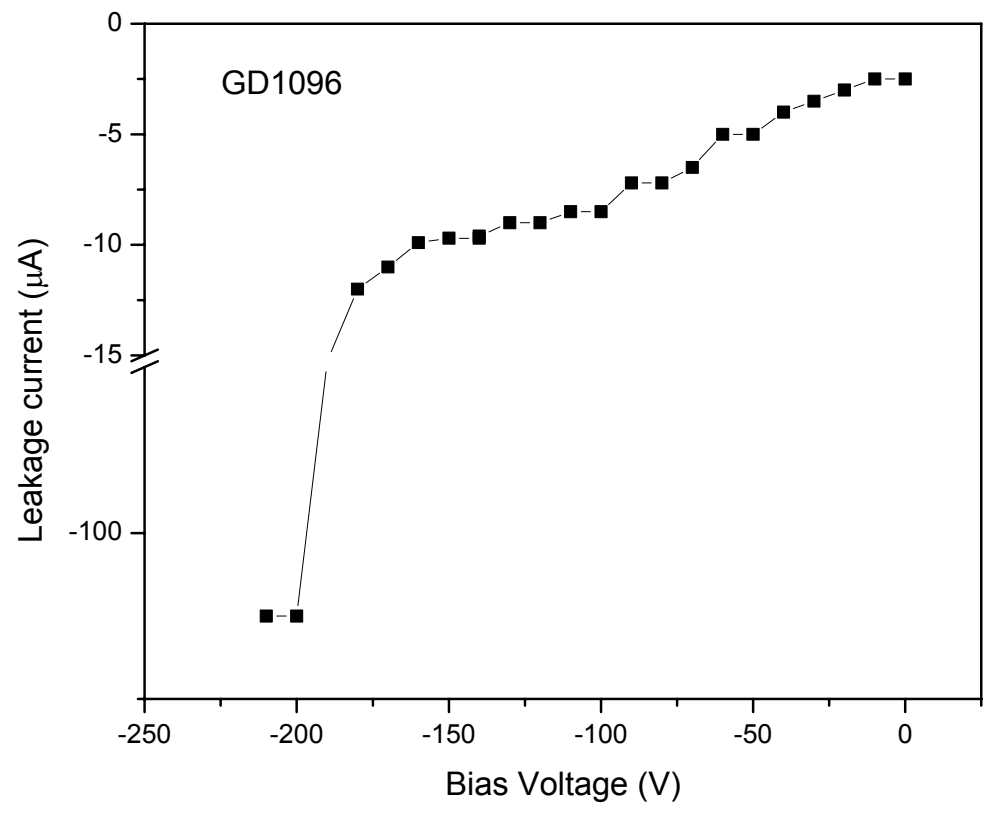

Fig. 3 Leakage current at different bias voltage for sample \# GD1096. 
GD1098:

In this case we tried a higher thickness of $12 \mu \mathrm{m}$ for i-layer. Also the two different sizes of the devices are tested: $0.25 \mathrm{~cm}^{2}$ and $0.5 \mathrm{~cm}^{2}$. The leakage current has been decreased to $5 \mu \mathrm{A}$ in dark for $0.5 \mathrm{~cm}^{2}$ and $0.3 \mu \mathrm{A}$ for $0.25 \mathrm{~cm}^{2}$ devices. The yield has been improved for this sample, for both on c-Si and SS. We also tested the devices under reverse bias in illuminated condition. Most of the devices responded low leakage current but still not satisfactory to achieve high signal to noise ratio (SNR). A photograph of the device is shown in Figure 4.

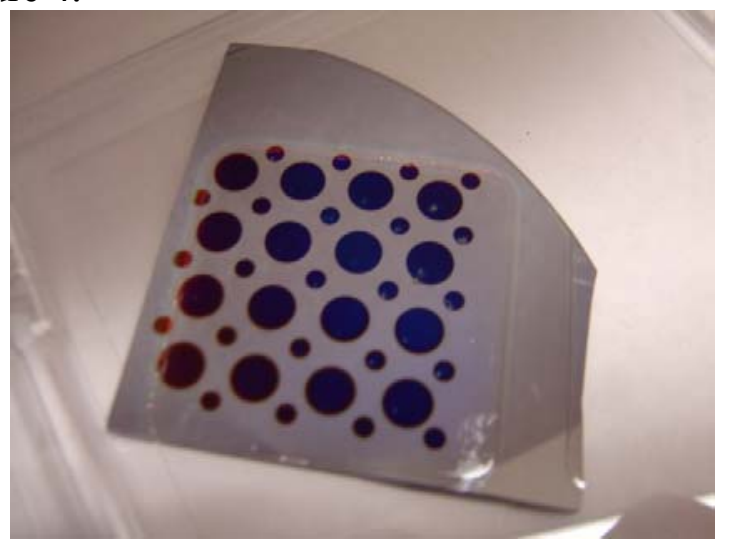

Fig. 4 Photograph of sample \#GD1098 with 0.25 and $0.05 \mathrm{~cm}^{2}$ device area.

We slightly varied the recipe (H-dilution) of i-layer deposition keeping the thickness constant. All the devices showed high yield and low leakage current (1-6 $\mu \mathrm{A}$ in dark) under high reverse bias in dark and illuminated condition. This sample promises high SNR due to its higher stability under reverse bias, we consider this sample for alpha particle measurement. Figure 5 shows the leakage current at different bias voltage for GD1099.

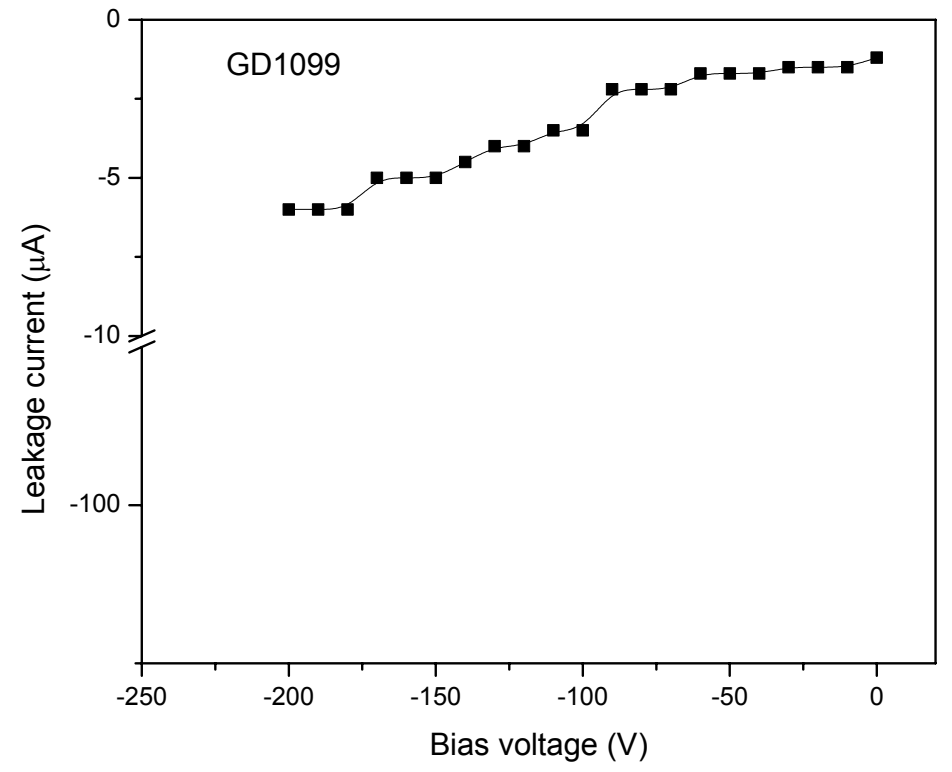

Fig. 5 Leakage current at different bias voltages for sample \# GD1099. 
GD1100:

We tried to increase the deposition rate of i-layer for this sample. No considerable improvement from the previous one has been noticed. We also consider this sample for alpha particle detection test.

GD1101:

In this sample we used much higher thickness for i-layer $(20 \mu \mathrm{m})$ and optimized the deposition recipe further to achieve better sticking and other requirements. The sample shows satisfactory yield (almost 100\%) accompanied with low leakage current. We consider this sample for alpha particle determination.

GD1130/GD1131:

So far only p-i-n structure of one diode has been developed and optimized in the previous samples. In these two runs we tried a $\mathrm{p}-\mathrm{i}-\mathrm{n} / \mathrm{n}-\mathrm{i}-\mathrm{p}$ structure of two reverse diodes for our purpose of development of pixilated detector with spatial resolution. Along with other insulating and conducting layers the structure comes to be as follows. c- $\mathrm{Si} / \mathrm{SiO}_{2} / \mathrm{Al}$ fingers (x-direction)/p-i-n (a-Si)/Al bias finger/n-i-p (a-Si)/Al fingers (y-direction) $/ \mathrm{SiO}_{2}$. A schematic diagram of the device structure is shown in Figure 6. A set of specially designed masks were required for deposition of different layers with different area, arrangement and shapes. Photographs of some of these masks shown in the Figure 7. In Figure 8 , a photograph of the device fabricated on c-Si substrate is shown on a measurement table in our laboratory.

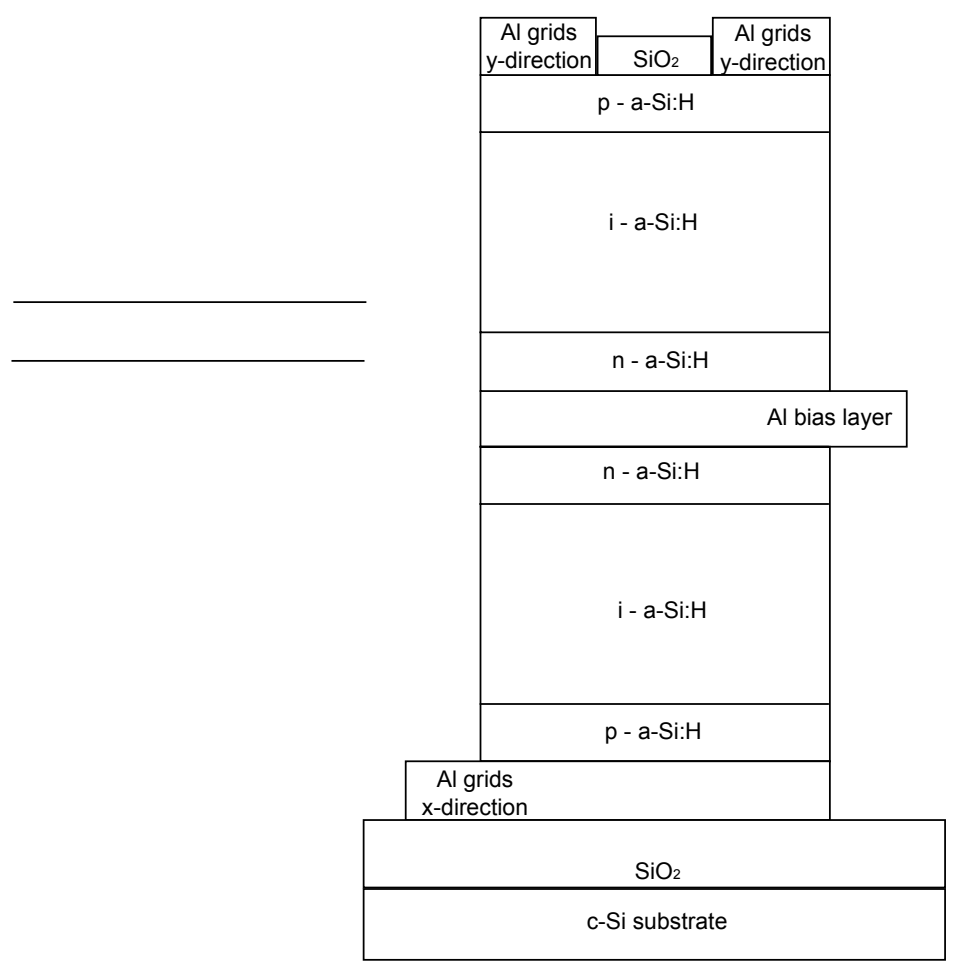

Fig. 6 Schematic diagram of sample \# GD1130/1131 with different semiconductor, insulating and conducting layers. 


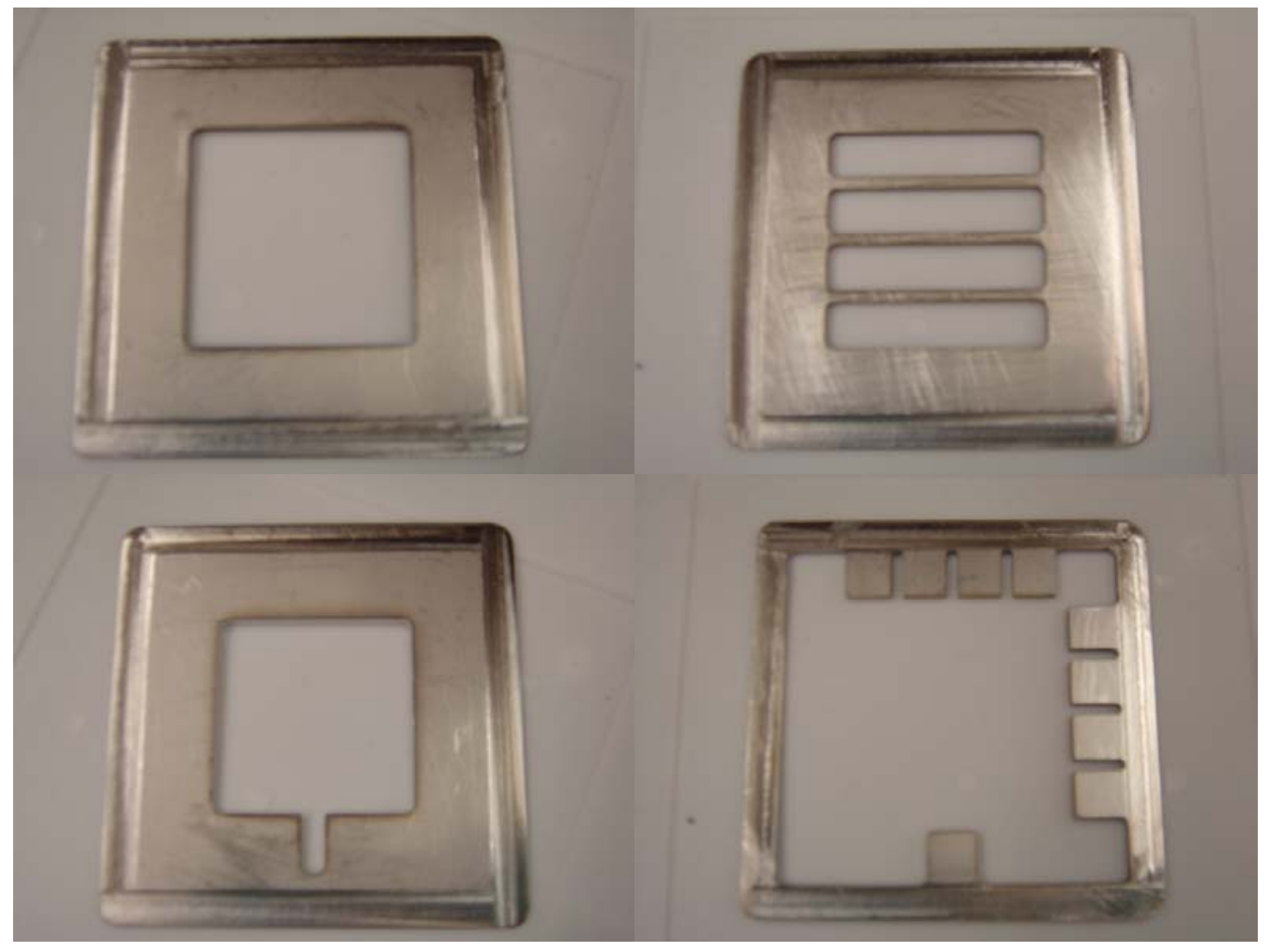

Fig. 7 Photographs of some masks used for deposition of a-Si, $\mathrm{Al}$ and $\mathrm{SiO}_{2}$ layers at different steps of the fabrication process

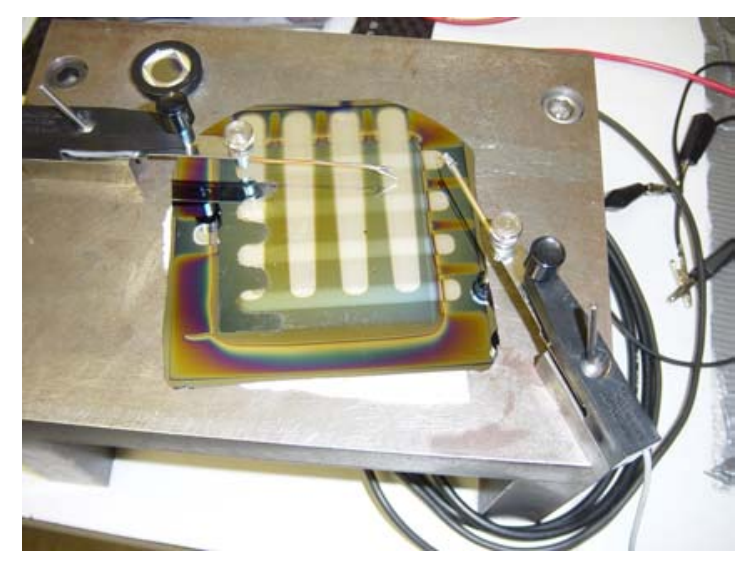

Fig. 8 Photograph of sample \# GD1130/1131 fabricated on c-Si showing pixilated structure. 
This sample preparation consists of eleven thin film layers of insulator, conductor and semiconductor materials by PECVD and sputtering processes on a substrate of size 4'x4'. We deposited each a-Si diode layers with a thickness of $10 \mu \mathrm{m}$ and deposited Al layers with specially designed fingers for proper electrical connections and biasing the devices. We did not get success of fabrication reverse operating diodes with this sample. The problem associated with this failure are peeling-off of the sample due to high a-Si thickness along with the combination of other type of layers and consequent short-circuit between $\mathrm{x}$-direction $\mathrm{Al}$ fingers and $\mathrm{Al}$ bias layer. We considered this problem and planned to reduce the thickness of each a-Si diode layer to $6 \mu \mathrm{m}$ in the following sample.

GD1132/GD1133:

In this sample with reduced thickness of a-Si layers, we did not get rid of peeling problem totally. We lost the middle bias Al layer due to peeling off. However, we were able to measure to the leakage current with applying $+30 \mathrm{~V} /-30 \mathrm{~V}$ bias on $\mathrm{x}$ and $\mathrm{y} \mathrm{Al}$ fingers. We observed a high leakage current for this sample and suspect some interdiffusion of intermediate Al layers through Si layers and proposed to isolate $\mathrm{Al}$ and $\mathrm{Si}$ layers with a layer of $\mathrm{ZnO}$ in the next run. However, we selected this sample for alpha detection measurement.

GD1134:

In this sample the layer structure is as follows: c-Si $/ \mathrm{SiO}_{2} / \mathrm{Al}$ finger (x-direction) $/ \mathrm{ZnO} / \mathrm{n}$-i$\mathrm{p}$ (a-Si)/Al fingers (y-direction). We tried to take a close look on a single diode first in this sample. In the pixilated structure one row is found to be short-circuited revealed in the leakage current measurement. This faulty row has been electrically isolated from the other part of the device and then checked with reverse bias-leakage current measurement. No such noticeable improvement in magnitude of leakage current occurred. We suspect the $\mathrm{SiO}_{2}$ layer to be not performing as expected. A photograph of the sample is shown in Figure 9.

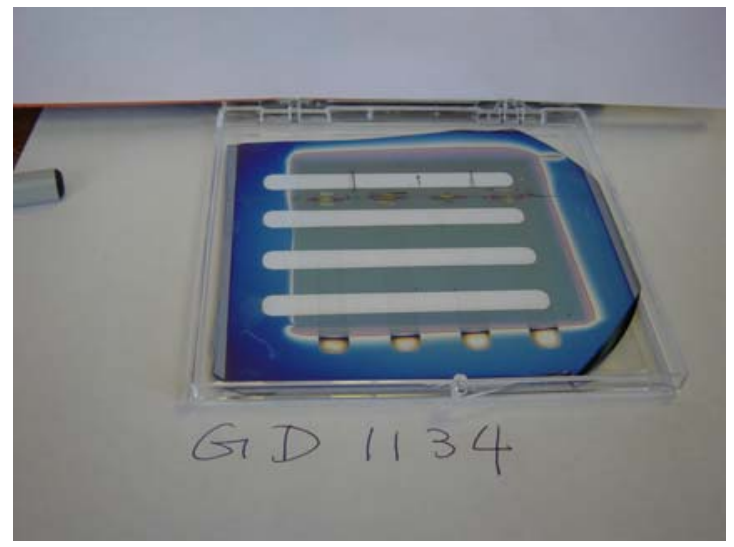

Fig. 9 Photograph of sample \# GD1134. 


\section{GD1136:}

Instead of using c-Si as substrate, we now choose glass, since there is no requirement of an insulating layer on glass for electrical insulation between $\mathrm{x}$-direction $\mathrm{Al}$ fingers. The structure of this sample became glass/Al fingers (x-direction)/p-i-n (a-Si)/Al fingers (ydirection). The Si layer thickness was kept at $6 \mu \mathrm{m}$. However, this sample has been revealed as totally short-circuited and also we found some peeling problem from the glass in this case. A photograph is shown in Figure 10.

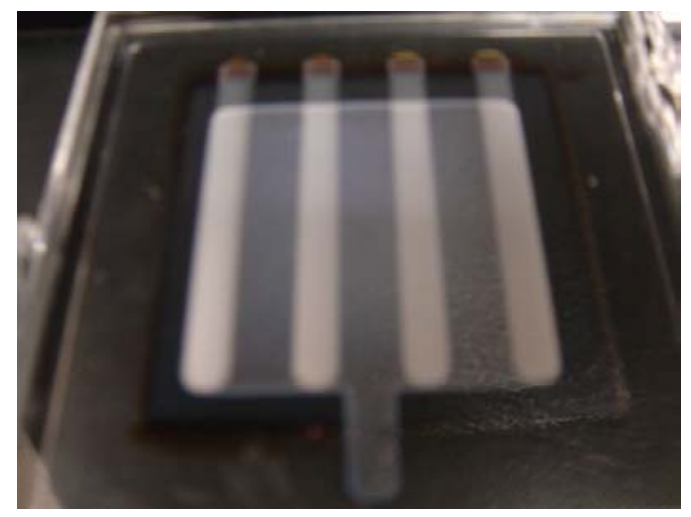

Fig. 10 Photograph of sample \# GD1136 fabricated on glass.

GD1135/GD1137:

To get rid of the short-circuit problem arising out of improper insulation layers, we explored a new structure where the substrate could be used as an electrical contact layer. The structure uses SS as the substrate and develops as follows: SS/n-i-p (a-Si)/Al fingers (x-direction)/n-i-p (a-Si)/Al fingers (y-direction). The device showed reliable stability under reverse bias and low leakage current, we select this sample to be investigated as alpha particle detector. A photograph of the sample is shown in Figure 11.

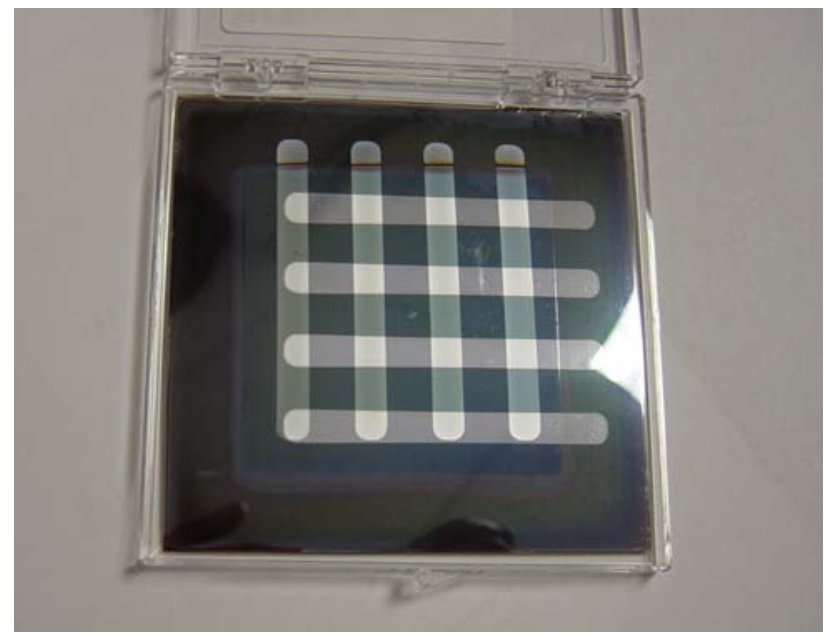

Fig. 11 Photograph of GD1135/1137 fabricated on stainless steel (SS) showing pixilated structure. 
GD1138/GD1140:

In the previous sample a thickness of $6 \mu \mathrm{m}$ was used for the Si layer which was intended to increase in this sample. Each Si diodes were deposited with $10 \mu \mathrm{m}$ thickness. We obtained a sample with slight peeling, however the leakage current seemed to be still low in this sample also.

GD1139/GD1141:

Again we tried another sample on glass and taken sufficient care by inserting $\mathrm{ZnO}$ intermediate layers in between $\mathrm{Al}$ and $\mathrm{Si}$ layers. The structure was as follows: glass/Al fingers (x-direction)/ZnO/p-i-n (a-Si)/ZnO/Al bias layer/ZnO/n-i-p (a-Si)/ZnO/Al fingers (y-direction). This device structure was also tested under reverse bias for leakage current determination and found to have high leakage current in spite of the incorporation of $\mathrm{ZnO}$ layers.

\section{GD1144/GD1145:}

A new structural arrangement for the electrical contacts has been made for this sample. We tried this sample on SS with a specially arranged four separate sectors of interconnected Al grids on 4'x4' area. A sketch of the structure is shown in Figure 12 and one of the four quarters of the sample is shown in Figure 13.

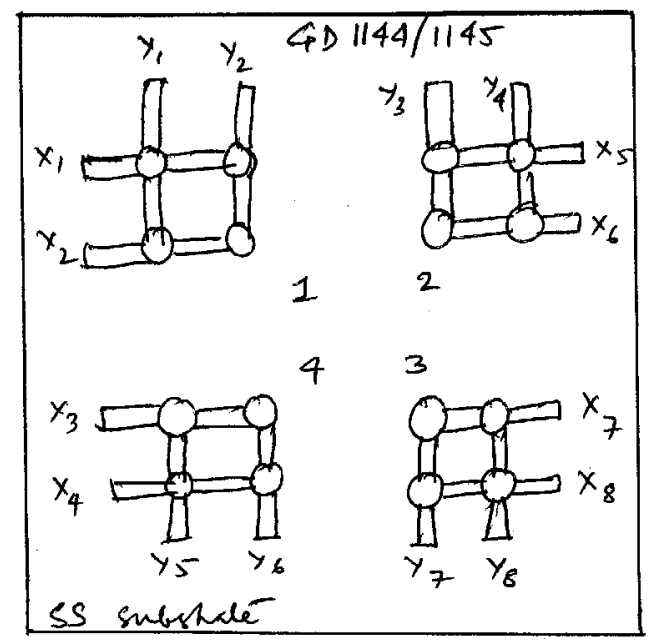

Fig. 12 A sketch of the Al grid arrangement on silicon diodes as contacts with four quarters on 4'x4' area for sample \# GD1144/1145. 


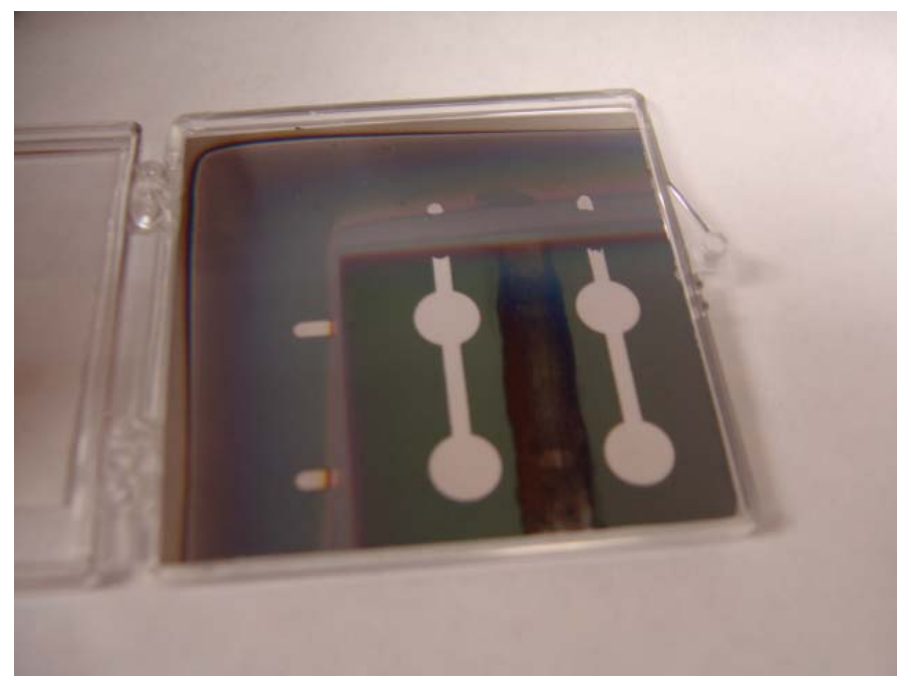

Fig. 13 A photograph of one of the four quarters of sample \#GD1144/1145 with special Al grids on it. Some portions of the grid were etched out to separate shorted

\section{(c) Detection of alpha particles}

The test for alpha particle detection has been done in parallel in Toledo (MWOE/UT) and in Oak Ridge (Oak Ridge National Lab, ORNL). The following describes the test results done in both the places.

The alpha particle detection station in Toledo is shown in Figure 14. The alpha source has been mounted on top attached to the lid. The sample has been mounted on a insulating board (planar) so that the source could focus the radiation normally on the sample. All the electrical connections and electronics are kept under the sample mounting board.

At UT, we tested samples under alpha particle radiation and counted the signal intensity with and without radiation. With a reverse bias of $-200 \mathrm{~V}$, we obtained detectable signal from the device GD1110. For better contact we coated a thin layer of gold $(\mathrm{Au})$ on top of the each small area devices. A photograph of sample GD1099 with gold contact layer is shown in Figure15. The results of the detection is described in table2.

Table 2: Results of alpha particle detection for GD1101.

\begin{tabular}{|c|c|c|c|c|c|}
\hline Sample & Bias (V) & Gain & Noise level (mV) & \multicolumn{2}{|c|}{ Counts } \\
\cline { 4 - 6 } & & & & Source ON & Source OFF \\
\cline { 3 - 6 } GD1101 & -200 & 100 & 200 & 3560 & 230 \\
\cline { 2 - 6 } & -200 & 250 & 200 & 5050 & 170 \\
\hline
\end{tabular}



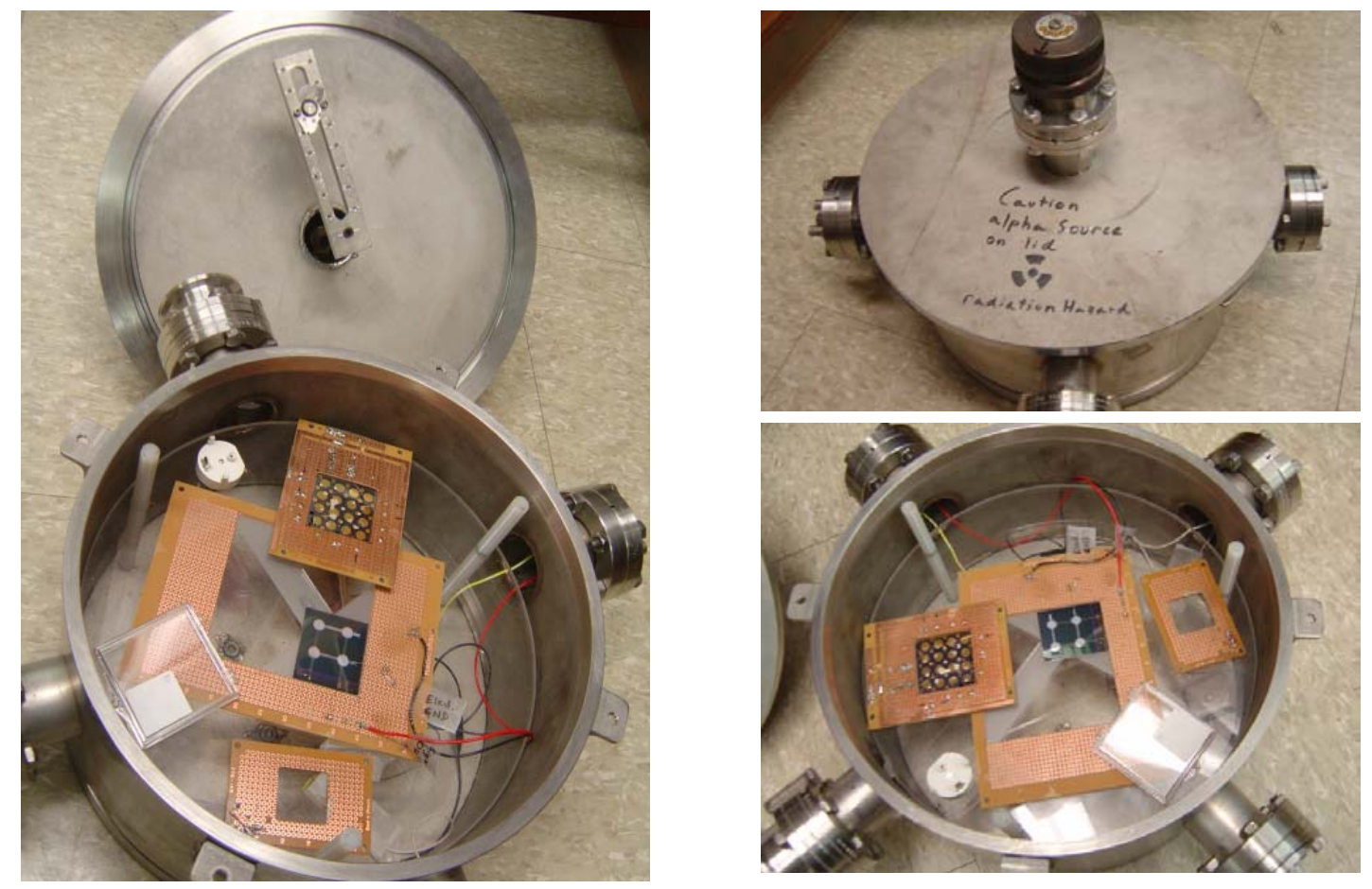

Fig. 14 Photograph of the UT alpha detection station showing (a) source on lid and the mounted sample and measurement unit at (b) closed and (c) open position.

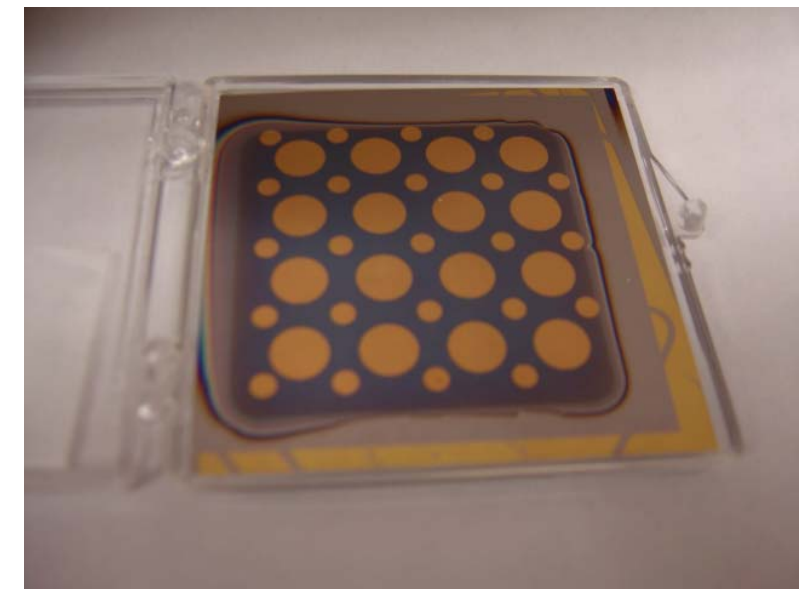

Fig. 15 A photograph of sample \# GD1101 with gold coating as top contact used for alpha detection test. 
At ORNL, we obtained signal from GD1099 and GD1110 also. We obtained 3:1 to 5:1 SNR from GD1099 and 3:1 from GD1101. For GD1101 the count rate is 10\% higher than that of GD1099 where the count is between 4500 and 4800 per second. The noise level was found to be as low as $75-100 \mathrm{mV}$.

The results at ORNL on the sample GD1099 SS $(12 \mu \mathrm{m})$ are:

Device \# $23100 \mathrm{mV}$ noise, $250 \mathrm{mV}$ signal, breakdown Voltage is 310, count rate is 4821 counts/sec through a $4 \mathrm{~mm}$ hole (through the contact)

Device \# $32 \quad 75 \mathrm{mV}$ noise, $250 \mathrm{mV}$ signal, -200V bias

Device \# $3375 \mathrm{mV}$ noise, $250 \mathrm{mV}$ signal, -200V bias

Device \# $3475 \mathrm{mV}$ noise, $250 \mathrm{mV}$ signal, -200V bias

Device \# $41100 \mathrm{mV}$ noise, $500 \mathrm{mV}$ signal, -200V bias.

On sample GD1101, separately at ORNL, we obtained good signal also. GD1101 on SS: Device\#32 produces, $140 \mathrm{mV}$ noise, $400 \mathrm{mV}$ signal, (Low voltage breakdown at bias $-90 \mathrm{~V}$ ) Count-rate is $10 \%$ higher that the $12 \mu \mathrm{m}$ sample GD1099.

The pulse height spectrum of GD1099 measured at ORNL is shown in Figure 16.

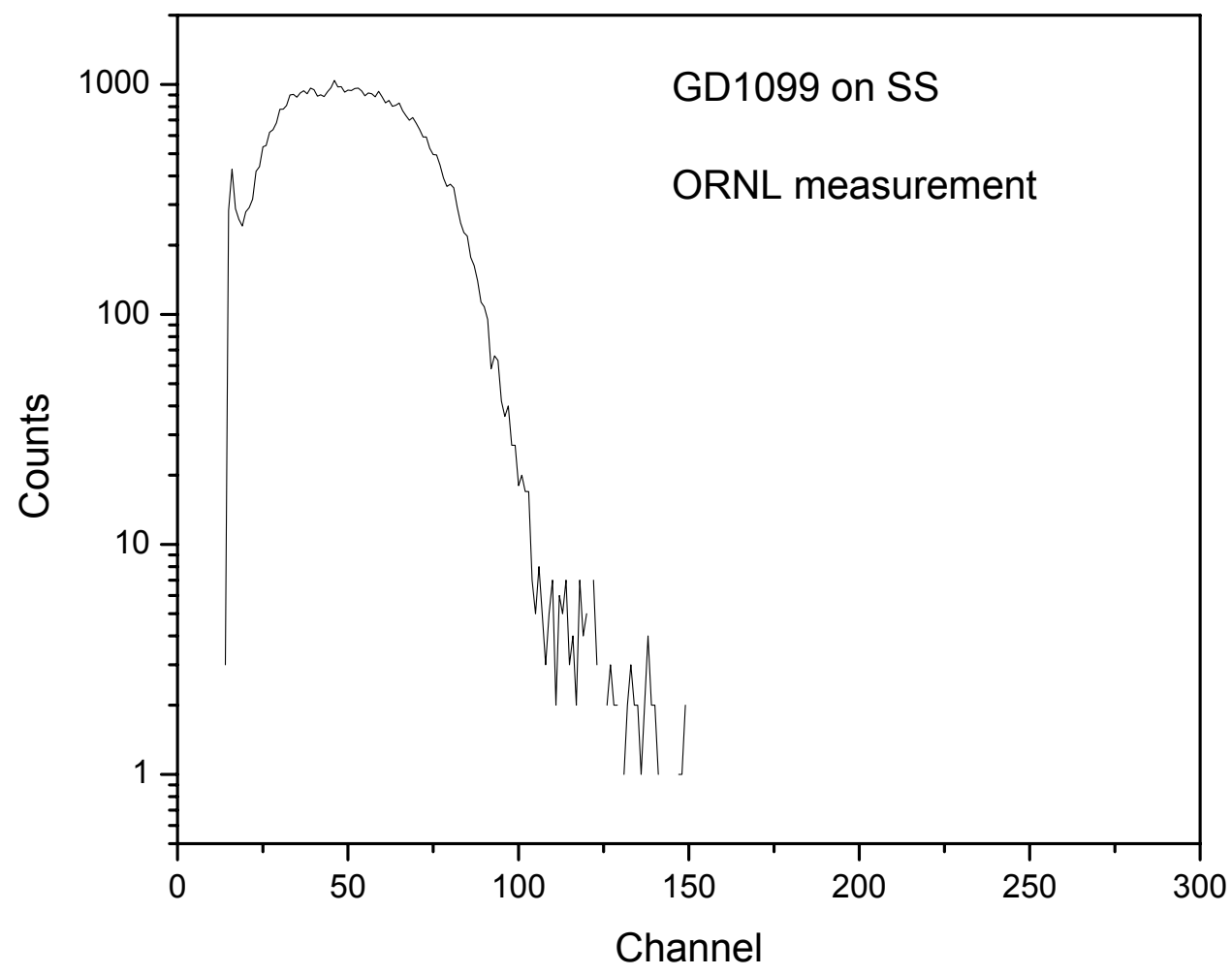

Fig. 16 Pulse height spectrum of sample \# GD1099 measured at ORNL. 
The same test on pixilated samples GD1134 failed due to high noise level ( 1V) at $-100 \mathrm{~V}$ reverse bias. We used the devices GD1137/GD1137 and GD1136 for alpha particle detection, however the noise level itself was very high $(1-2 \mathrm{~V})$. To minimize the noise we tried to apply Indium at the contacts (with alligator clip), but did not reduce the noise. We were not able to solder indium with Al tabs. In case of GD1138/GD1140, though the leakage current found to be of the order of microamps $(\mu \mathrm{A})$ for the good diodes, we did not see any signal in alpha particle detection and noise level was still high (1V). In GD1139/GD1141, the diodes were found as very unstable (even with -30V) and the leakage current was very high (several $\mathrm{mA}$ ). Very high noise level $(2 \mathrm{~V})$ and no detection of alpha particle result from this trial. In GD1144/GD1145, due to its unique Al grids structure, we mechanically cut and separate the 4'x4' sample into four quarters. The samples were found to show reasonable open circuit voltage under weak illumination and low leakage current ( in the $\mu \mathrm{A}$ ranges). The diodes were stable enough (tested up to $150 \mathrm{~V})$. Still, we did not find any signal from alpha detection, the noise level was at $1 \mathrm{~V}$.

\section{Neutron detection}

The neutron detection tests were done at ORNL with the samples in which we found signals from alpha particle radiation.

For neutron detection, we painted the sample surface with a thin layer of Boron Nitride paint atop the devices. However, we failed to see neutrons with a moderated AmBe neutron source having a source flux of greater than $10^{7}$ neutrons $/ \mathrm{cm}^{2} / \mathrm{s}$. The source was at a distance of about $40 \mathrm{~cm}$ from the sample. 